\title{
USO DEL ANÁLISIS MULTIVARIADO FACTORIAL MIXTO PARA CARACTERIZAR VARIABLES CLIMÁTICAS Y EDÁFICAS EN SISTEMAS AGROPECUARIOS COLOMBIANOS
}

\author{
USING MULTIVARIATE MIXED FACTOR ANALYSIS TO \\ CHARACTERIZE CLIMATIC AND EDAPHIC VARIABLES IN \\ COLOMBIAN AGRICULTURAL SYSTEMS
}

\author{
Julián Andrés Castillo Vargas \\ Universidad Nacional Abierta y a Distancia UNAD \\ ORCID: https://orcid.org/0000-0001-5163-5127 \\ Email: andres.castillo@unad.edu.co \\ Wilmer Alfonso Cuervo Vivas \\ Universidad Nacional Abierta y a Distancia UNAD \\ ORCID: https://orcid.org/0000-0002-3240-0338 \\ Email: wilmer.cuervo@unad.edu.co
}

Citación: Vargas, J.A.C., y Cuervo, W. (2021). Uso del análisis multivariado factorial mixto para caracterizar variables climáticas y edáficas en sistemas agropecuarios Colombianos. Agricolae \& Habitat, 4(2), 06 - 19. DOI: https://doi.org/10.22490/26653176.4584

\section{RESUMEN}

Contextualización: La productividad de los sistemas agropecuarios se define como la combinación de las variables climáticas y edáficas que los caracteriza. Por tanto, el entendimiento de la interrelación de tales variables debe ser estudiado bajo un abordaje multivariado.

Vacío del conocimiento: Hasta ahora, no han sido desarrollados estudios que determinen la estructura multivariada de variables asociadas a climatología y edafología de los sistemas agropecuarios en Colombia.
Propósito: determinar la estructura multivariada de variables climáticas y edáficas en los sistemas agropecuarios colombianos usando la técnica de análisis multivariado factorial mixto (AMFM).

Metodología: Se construyó una base de datos con 167 registros de variables climáticas (altitud, temperatura y humedad relativa) y edáficas (textura del suelo, $\mathrm{pH}$, actividad enzimática de la ureasa y actividad enzimática de la catalasa) de predios destinados a la producción agrícola o pecuaria primaria en 21 departamentos y 120 municipios de Colombia. La estructura de las 
variables anteriormente mencionadas fue analizada usando un AMFM utilizando el software Statistica, versión 7.0, siendo estas clasificadas en factores latentes. Una variable era asociada a un factor latente, cuando el valor absoluto de su correlación con el factor era mayor que 0.5 .

Resultados y conclusiones: Usando el AMFM, se extrajeron cuatro factores latentes para las siete variables estudiadas, los cuales representaron un $75 \%$ de la varianza total. El primer factor se asoció positivamente con la altitud $(0,950)$ y negativamente con la temperatura $(-0,957)$, siendo denominado como "Climático". El segundo factor se correlacionó negativamente con la humedad relativa $(-0,790)$ y la actividad ureasa ($0,706)$. Adicionalmente, este tuvo una asociación negativa secundaria con el pH ($0,343)$, siendo denominado como "Ureasaagua- $\mathrm{pH}^{\prime}$. El tercer factor fue denominado como "Fisicoquímica-suelo" y se correlacionó positiva y fuertemente con la textura del suelo $(0,917)$. Variables como la humedad relativa $(-0,281), \mathrm{pH}(0,306)$ y actividad ureasa $(0,211)$, también tuvieron una contribución secundaria a este factor. El cuarto factor se correlacionó positivamente con el pH $(0,544)$ y la actividad catalasa $(0,887)$, siendo denominado como "Catalasa$\mathrm{pH}^{\prime \prime}$. Del presente estudio se puede concluir que el AMFM reveló la estructura multivariada de variables climáticas y edáficas asociadas a los sistemas de producción agropecuaria colombiana.

Palabras clave: actividad catalasa, actividad ureasa, análisis factorial mixto, $\mathrm{pH}$, suelo, temperatura

\section{ABSTRACT}

Contextualization: The productivity of agricultural systems is defined as the combination of climatic and edaphic variables that characterize them. Therefore, the understanding of the interrelation of such variables must be studied under a multivariate approach.
Knowledge gap: Until now, no studies have been developed determining the multivariate structure of variables associated with the climatology and edaphology of agricultural systems in Colombia.

Purpose: To determine the multivariate structure of climatic and edaphic variables in Colombian agricultural systems using the multivariate mixed factor analysis technique (AMFM).

Methodology: A database with 167 records of climatic (altitude, temperature, and relative humidity) and soil (soil texture, $\mathrm{pH}$, enzymatic activity of urease and enzymatic activity of catalase) variables of farms destined to primary agricultural or livestock production in 21 departments and 120 municipalities in Colombia was constructed. The structure of the aforementioned variables was analyzed using an AMFM by the software Statistica, version 7.0, and the variables were classified into latent factors. A variable was associated with a latent factor, when the absolute value of its correlation with the factor was greater than 0.5 .

Results and conclusions: Using the AMFM, it was possible to extract four latent factors for the seven studied variables, which represented $75 \%$ of the total variance. The first factor was positively and negatively associated with altitude (0.950) and temperature $(-0.957)$, respectively, being named as "Climate". The second factor was negatively correlated with relative humidity ($0.790)$ and urease activity $(-0.706)$. Also, this had a negative secondary association with $\mathrm{pH}(-0.343)$, being termed as "Ureasewater-pH". The third factor was denominated as "Physicochemical-soil" and it was positively correlated with the texture of the soil (0.917). In addition, variables such as relative humidity $(-0.281), \mathrm{pH}(0.306)$, and urease activity (0.211) also had a secondary contribution to this factor. The fourth factor was positively correlated with $\mathrm{pH}(0.544)$ and catalase activity (0.887), being named as "Catalase-pH". From the present study, it can 
be concluded that the AMFM revealed the multivariate structure of climatic and edaphic variables associated to Colombian agricultural production systems.
Keywords: catalase activity, factor mixed analysis, $\mathrm{pH}$, soil, temperature, urease activity

\section{RESUMEN GRÁFICO}
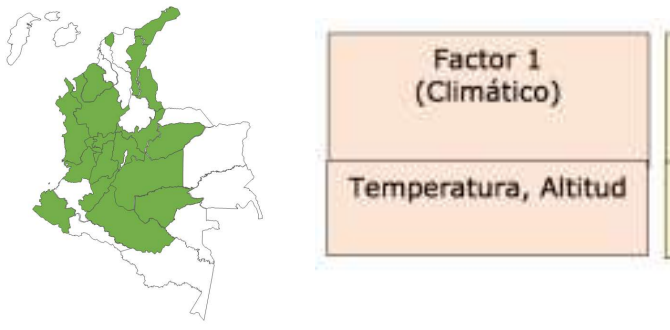

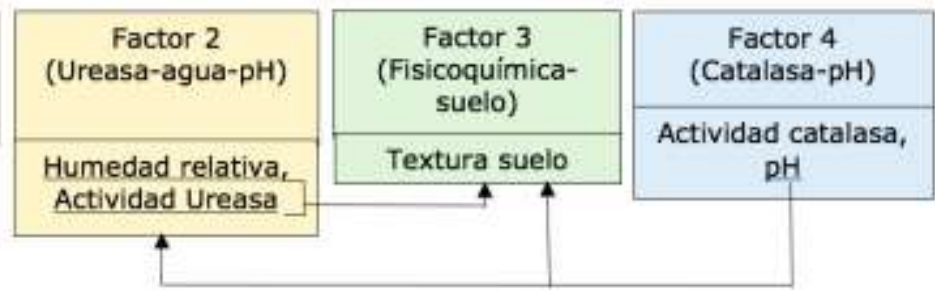

Fuente: autores

\section{INTRODUCCIÓN}

La productividad animal y vegetal de los sistemas agropecuarios puede ser representada como el resultado de la combinación de las variables climáticas y edáficas que los caracteriza (Agnusdei, 2013). Paralelamente se sabe, que las condiciones climáticas y edáficas están interrelacionadas, afectando esta interrelación la producción de recursos vegetales en los sistemas de producción agropecuarios (Mogollón et al., 2016). Esto inherentemente lleva a pensar, que un abordaje simultáneo de las respuestas o tendencias de variables climáticas y edáficas podría proporcionar una visión más realista y práctica para la modelación y entendimiento de las respuestas de sistemas de producción animal y vegetal.

Diversos estudios han evaluado el efecto de factores físicos, químicos y bioquímicos del suelo, así como de las condiciones climáticas sobre la productividad de los sistemas agropecuarios (Vallejo-Quintero, 2013; Mogollón et al., 2016). Sin embargo, hasta ahora, no han sido desarrollados estudios que determinen la estructura multivariada de variables asociadas a climatología y edafología de los sistemas agropecuarios en Colombia. Para este fin, el uso de técnicas de estadística multivariada podría ser lo más adecuado, ya que esta rama de la estadística se encarga del análisis simultaneo de variables en sistemas (Johnson y Wichern, 2014). Entre la gama de técnicas asociadas a la estadística multivariada, el análisis factorial es el más adecuado para entender la estructura de variables asociadas (Hair, 2014).

El análisis multivariado factorial mixto (AMFM) es una técnica de la estadística multivariada que es usada para descifrar la estructura múltiple variables cuantitativas y categóricas que componen los sistemas y permite determinar la covarianza máxima entre las variables originales (Snedecor y Cochran, 1989). Esta técnica divide la varianza total de un sistema multivariado en dos componentes: la varianza que comparten todas las variables (es decir, la comunalidad) y la varianza particular de cada variable (es 
decir, la singularidad; Johnson y Wichern, 2014). De esta forma, el objetivo de esta investigación fue determinar la estructura multivariada de variables climáticas y edáficas en sistemas agropecuarios colombianos, usando la técnica de AMFM.

\section{MATERIALES Y MÉTODOS}

\section{Conformación de la base de datos}

Se construyó una base de datos que contiene un total de 167 registros provenientes de la recolección de variables climáticas (altitud, temperatura y humedad relativa) y edáficas (textura del suelo, $\mathrm{pH}$, actividad enzimática de la ureasa y actividad enzimática de la catalasa) entre 2017 y 2018 de predios destinados a la producción agrícola o pecuaria primaria a lo largo de 21 departamentos (Antioquia, Atlántico, Boyacá,
Caldas, Caquetá, Casanare, Cauca, Cesar, Chocó, Córdoba, Cundinamarca, Guajira, Guaviare, Huila, Meta, Nariño, Norte de Santander, Quindío, Risaralda, Tolima y Valle) y 120 municipios de Colombia ( $\mathrm{n}=$ 167; Tabla 1; Figura 1). La recolección de las variables climáticas fue realizada a partir de informaciones publicadas por el Instituto de Hidrología, Meteorología y Estudios Ambientales (IDEAM, 2021) y la recolección de las variables edáficas, fue hecha por estudiantes de los programas de Zootecnia y Agronomia (Curso: Bioquímica metabólica), de la Universidad Nacional Abierta y a Distancia en su localidad, bajo supervisión y protocolos estandarizados, los cuales se detallan en esta sección. Cada registro fue revisado en términos de trazabilidad y calidad por parte de los autores, antes de ser incluido en la base de datos de estudio.

Tabla 1. Distribución de frecuencias de las variables cualitativas y estadística descriptiva de variables cuantitativas usadas en el análisis.

\section{Variables cualitativas No. municipios}

Textura del suelo

$\begin{array}{ll}\text { Arcilloso } & 16 \\ \text { Arenoso } & 15\end{array}$

Franco arcilloso 62

Franco arenoso $\quad 55$

Limoso 19

$\mathrm{pH}$

$\begin{array}{rc}{[1,0-5,9]} & 55 \\ (5,9-10,9] & 110 \\ (10,9-14) & 2\end{array}$

Actividad Ureasa

$\begin{aligned} \text { Baja } & 72 \\ \text { Media } & 65 \\ \text { Alta } & 30\end{aligned}$

Actividad Catalasa

$\begin{aligned} \text { Baja } & 57 \\ \text { Media } & 68 \\ \text { Alta } & 42\end{aligned}$




\begin{tabular}{ccccc}
\hline Variables cuantitativas & Media & S & Mínimo & Máximo \\
\hline Altitud $(\mathrm{msnm})$ & 1273,7 & 872,3 & 4,0 & 3125,0 \\
Temperatura $\left({ }^{\circ} \mathrm{C}\right)$ & 22,6 & 5,4 & 7,0 & 34,0 \\
Humedad relativa $(\%)$ & 70,6 & 14,3 & 9,0 & 94,0 \\
\hline
\end{tabular}

Fuente: autores

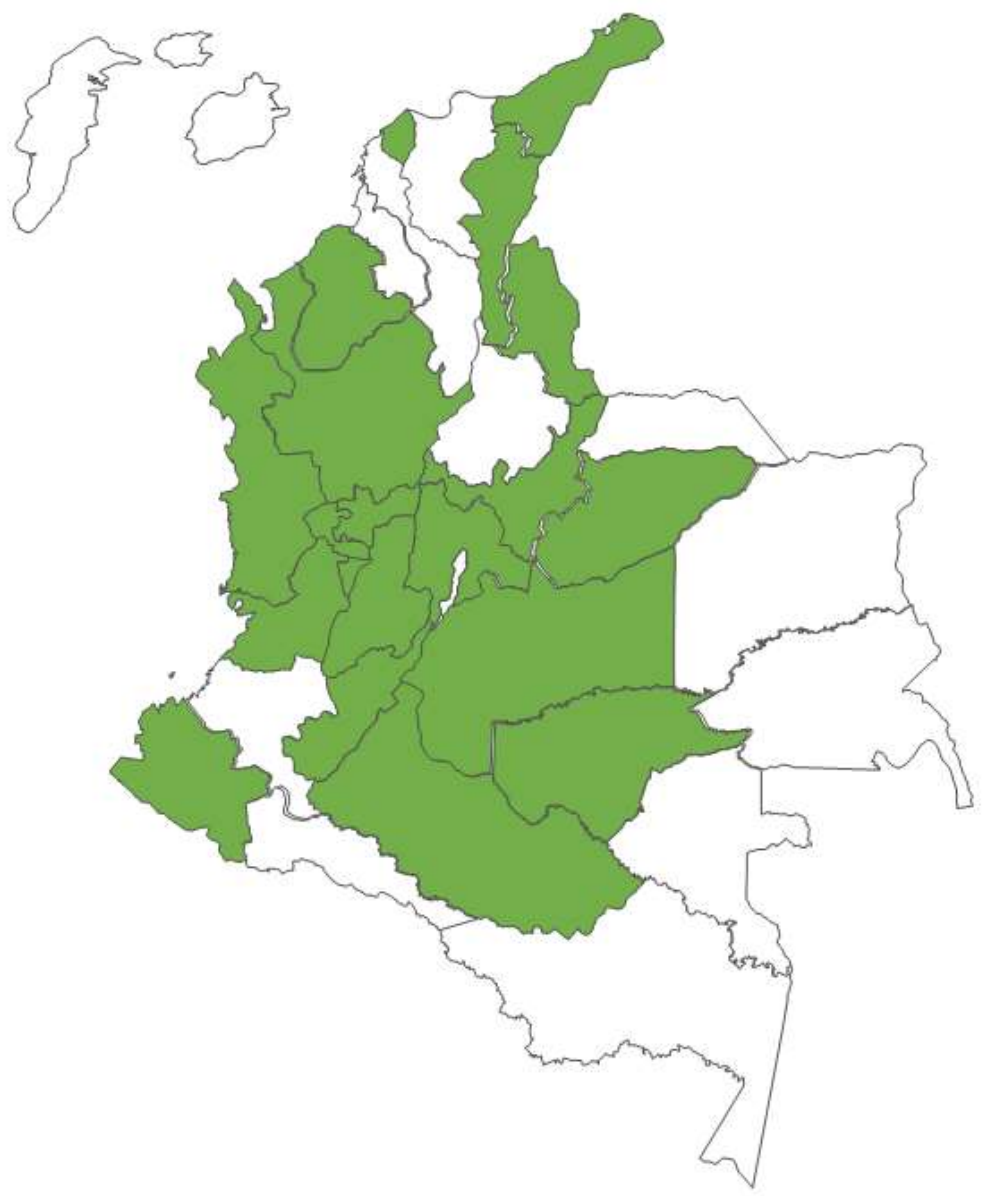

Figura 1. Distribución geográfica de los departamentos de Colombia incluidos en el estudio. Fuente: autores

Las variables denominadas como (baja; media; alta) y actividad enzimática de cuantitativas continuas fueron: altitud la catalasa en el suelo (baja; media; alta). (msnm), temperatura $\left({ }^{\circ} \mathrm{C}\right)$ y humedad relativa (\%) y las cualitativas o categóricas fueron: textura del suelo (arcilloso; arenoso; franco arenoso; limoso), $\mathrm{pH}$ (bajo: [1,0 5,9]; medio: $(5,9-10,9]$; alto: $(10,9-14))$, actividad enzimática de la ureasa en el suelo

\section{Determinación de variables}

Geo-referenciación del predio. Por medio de la aplicación Google Maps ${ }^{\circledR}$ de la plataforma Google ${ }^{\circledR}$, se identificó la ubicación de la vereda $y$ el municipio del predio a 
caracterizar. En la totalidad de los datos seleccionados, se realizó un doble chequeo con la información oficial del sitio web de la alcaldía de cada municipio.

\section{Variables climáticas}

Para la estimación y toma de los datos, se recurrió a la información climatológica del Instituto de Hidrología, Meteorología y Estudios Ambientales (IDEAM, 2021), siguiendo la ruta de la opción Meteorología Climatología - Archivo de Climatología, la cual contiene el reporte de temperatura máxima, mínima y promedio, como también de humedad y precipitación a escala mensual y a nivel municipal.

Asi mismo, la información colectada se cotejó con la información disponible en sitios web especializados en el reporte en tiempo real del promedio de temperatura y otras variables climatológicas como precipitación y humedad relativa, usando AccuWeather (AccuWeater, 2021; Inc, Pensilvania, EU) y Climate Services Partnership (CSP, 2021; Columbia, SC, EU).

\section{Variables edáficas}

Determinación del $\mathrm{pH}$. Se tomaron $100 \mathrm{~g}$ de suelo y se mezclaron con $500 \mathrm{~mL}$ de agua potable. Dicha mezcla se agitó durante 20 minutos de manera continua y posteriormente se dejó en reposo por una hora en decantación. A partir del sobrenadante resultante, se realizó la estimación del $\mathrm{pH}$ utilizando cintillas comerciales de $\mathrm{pH} O$ por medio de potenciómetro (de acuerdo con la disponibilidad en la zona donde se realizó la determinación).

Caracterización de la textura - Método indirecto por integridad de agregados. Teniendo como base la metodología sugerida por el servicio de extensionistas de estados Unidos, para evaluación de la textura y de la actividad biológica de suelos (USDA, 1999), se realizó la evaluación de la textura del suelo, mediante el siguiente procedimiento: se tamizaron 6 muestras de suelo de $100 \mathrm{~g}$ cada una, las cuales fueron humedecidas superficialmente y de manera gradual, para ser mezcladas y formar agregados cilíndricos que fueron secados al sol durante 1 hora. Posteriormente, los agregados fueron sumergidos en recipientes con agua y se observó durante $5 \mathrm{~min}$ su desagregación, luego de lo cual, los agregados fueron retirados con una cuchara plástica.

Se asignaron valores altos para suelos arenosos (más de la mitad de la integridad estructural del suelo se perdía al cabo de 5 segundos de inserción en agua). Valores medios para suelos limosos (en las muestras en las que aproximadamente la mitad de la integridad estructural del suelo se perdía al cabo de 5-30 segundos de inmersión) y valores altos para suelos arcillosos (en las muestras en las que la mitad o menos de la integridad estructural se perdía al cabo 30 300 segundos de inmersión).

Caracterización de la textura - Método indirecto por decantación. Se tomaron $120 \mathrm{~g}$ de suelo que fueron homogenizados manualmente; posteriormente, fueron tamizados y solubilizados con agua potable, hasta lograr un volumen de $250 \mathrm{~mL}$ en una botella de vidrio, en la que se realizó la lectura a simple vista de los decantados. La botella tapada incluyendo la mezcla, fue agitada manualmente 10 veces durante 2 minutos cada vez, luego de lo cual se dejó reposar durante 24 horas, para posteriormente observar las diferentes fracciones decantadas en la solución del suelo. Se midió la altura $(\mathrm{cm})$ de la solución 
en la botella de vidrio con una regla y al mismo tiempo se marcaron las fases en las cuales se separaron los decantados del suelo. Esta proporción indicó de arriba hacia abajo en la botella, la cantidad aproximada de arcillas, limos y en el fondo arena arcillas. Las proporciones con respecto a la totalidad de la solución en la botella, se utilizaron para estimar la textura de la muestra de suelo.

Estimación de la actividad ureasa. La actividad de la enzima ureasa en suelo, se determinó de forma cualitativa, usando la metodología propuesta por Arreaza et al. (2002): se realizó una mezcla de partes iguales de frijol canavalia (Canavalia ensiformis) molido y disuelto en agua (extracto de ureasa) con urea comercial. Como control positivo se tomó una muestra de frijol molido a la que se le agregó en igual medida urea comercial. Luego, como muestra experimental se tomó una muestra de $100 \mathrm{~g}$ de suelo a la que se le agregó igual cantidad de urea, mezcla que se disolvió en una proporción de 1 a 5 con agua. El desprendimiento de amonio detectado de forma sensorial (olor intenso, medio y leve), sirvió como indicador cualitativo de la presencia y grado (alto, medio o bajo, respectivamente) de actividad ureasa.

Estimación de la actividad catalasa. Se realizó la determinación cualitativa de actividad catalasa, utilizando la metodología propuesta por Gaete et al. (2010), con modificaciones: Teniendo en cuenta la elevada actividad de la enzima catalasa en los peroxisomas de los hepatocitos, se utilizó hígado de pollo macerado como control positivo de la actividad catalasa. Para esto, $20 \mathrm{~g}$ de hígado de pollo fueron macerados y mezclados con $25 \mathrm{~mL}$ de peróxido de hidrogeno $\left(\mathrm{H}_{2} \mathrm{O}_{2}\right)$ comercial. De esta mezcla, se registró la cantidad de espuma desprendida. De igual manera, a $25 \mathrm{~g}$ de suelo, se le adicionaron 25 $\mathrm{mL}$ de $\mathrm{H}_{2} \mathrm{O}_{2}$ y se registró la altura de la espuma producida, categorizándola como alta (más de $10 \mathrm{~mm}$ ), media (5 - $9 \mathrm{~mm}$ ) o baja (menos de $5 \mathrm{~mm}$ ).

\section{Análisis estadístico}

Se realizó un análisis multivariado factorial mixto (AMFM) utilizando el software Statistica, versión 7.0 (Stat Soft Inc, Tulsa, OK, EE. UU.), para explorar la estructura multivariada de las variables climáticas y edáficas en los sistemas de producción agropecuarios colombianos (Johnson and Wichern, 2014). El objetivo principal de AMFM es explicar la (co) varianza de un sistema definido por $n$ variables medidas $\left(Y_{1}\right.$, $\left.\ldots, Y_{n}\right)$ al generar un número menor $p(p<n)$ de variables no observables latentes $\left(X_{1}, \ldots\right.$, $\left.X_{p}\right)$, denominadas como factores latentes comunes. El AMFM acepta que la varianza de cada variable original puede descomponerse en sus componentes comunes (es decir, comunalidad) y únicos (es decir, unicidad). El modelo factorial descompone la matriz de covarianza de las variables medidas (Q) de la siguiente manera:

$$
Q=A A^{\prime}+\Phi(\text { ecuación } 1)
$$

donde $A A^{\prime}$ y $\Phi$ son las matrices de (co) varianza de comunalidad y unicidad, respectivamente (Snedecor and Cochran, 1989; Hair, 2014).

Como se indica en el modelo de (co)varianza, las variables medidas se pueden representar como una combinación de $\mathrm{p}$ factores comunes $(X)$ no observables más una variable única $(e)$ :

$$
\begin{aligned}
& y_{1}=a_{11} x_{1}+\ldots+a_{1 p} x_{p}+e_{1} \text { (Ecuación 2) } \\
& y_{n}=a_{n 1} x_{1}+\ldots+a_{n p} x_{p}+e_{n} \text { (Ecuación 3), }
\end{aligned}
$$


donde: a son las cargas que cuantifican la correlación entre el factor $p$ latente y la variable medida. Las cargas son los elementos de la matriz A (ecuación 1) del modelo de variación teórica.

La factibilidad de aplicación de la tecnica de AMFM en el banco de datos usado, se evaluó a partir del logaritmo en base 10 del determinante de la matriz de correlaciones y como criterio de elección del número de factores, se usó la recomendación propuesta por Kaiser (1958), la cual sugiere que factores con valor propio mayores o iguales a 1 , deben ser conservados en el análisis factorial. El número de factores a extraer se basó también en su legibilidad en términos de la relación con las variables originales y la cantidad de varianza explicada.

La legibilidad de cada factor se mejoró a través de una rotación Varimax Normalizada. Para extraer los factores latentes, se consideró que una variable estaba asociada con un factor latente específico, si el valor absoluto de su correlación con el factor específico era mayor o igual a 0.5 (Hair, 2014).

Adicionalmente, se realizó una regresión lineal entre la temperatura $\left({ }^{\circ} \mathrm{C}\right)$ y la altitud (msnm) de todas las ubicaciones geográficas evaluadas usando el método de los mínimos cuadrados, mediante el software Microsoft Excel ß. Se exploró tanto la normalidad de los residuales como la presencia de valores extremos (outliers) mediante un histograma de residuos construido en el programa mencionado.

\section{RESULTADOS Y DISCUSIÓN}

\subsection{RESULTADOS}

Los municipios evaluados en el análisis presentaron principalmente suelos franco arcilloso y franco arenoso, con un $\mathrm{pH}$ mayoritariamente entre 5,9 y 10,9, y con actividades ureasa y catalasa principalmente baja y media (Tabla 1). La mayor variabilidad entre municipios se registró en la altitud ( $C V=68,5 \%)$ y la menor en la humedad relativa $(\mathrm{CV}=20.2 \%)$.

El análisis multivariado factorial fue capaz de extraer cuatro factores latentes de las siete variables analizadas (Tabla 2 ), representando el $75,2 \%$ de la varianza total. El primer factor latente representó el $27,5 \%$ de la varianza total, y se asoció de forma positiva con la altitud y negativa con la temperatura. Por lo tanto, se le denominó como "Climático" (Tabla 2). El segundo factor latente explicó el $19,1 \%$ de la varianza total y principalmente se correlacionó de forma negativa con la humedad relativa y la actividad ureasa. Sin embargo, este factor tuvo una asociación secundaria con el pH. Por tanto, se denominó como "Ureasa-agua-pH" (Tabla 2). El tercer factor latente se denominó como "Fisicoquímica-suelo". Éste representó el $14,7 \%$ de la varianza total y se correlacionó fuertemente $y$ de forma positiva con la textura del suelo. Sin embargo, variables como la humedad relativa, $\mathrm{pH}$ y actividad ureasa, tuvieron una contribución secundaria a este factor (Tabla 2). Finalmente, el cuarto factor explicó el 13,9\% de la varianza total y se correlacionó positivamente con el $\mathrm{pH}$ y la actividad catalasa, siendo denominado como "Catalasa-pH" (Tabla 2). 
Tabla 2. Patrón de factores rotados (F) y estadísticos del análisis multivariado factorial mixto.

\begin{tabular}{lcccc}
\hline \multicolumn{1}{c}{ Variable } & $\begin{array}{c}\text { F1 } \\
\text { Climático }\end{array}$ & $\begin{array}{c}\text { F2 } \\
\text { Ureasa- } \\
\text { agua-pH }\end{array}$ & $\begin{array}{c}\text { F3 } \\
\text { Fisicoquímica- } \\
\text { suelo }\end{array}$ & $\begin{array}{c}\text { F4 } \\
\text { Catalasa- } \\
\text { pH }\end{array}$ \\
\hline Altitud & $\mathbf{0 , 9 5 0}$ & 0,042 & 0,115 & 0,028 \\
Temperatura & $\mathbf{- 0 , 9 5 7}$ & $-0,020$ & 0,005 & $-0,076$ \\
Humedad relativa & $-0,039$ & $\mathbf{- 0 , 7 9 0}$ & $\underline{-0,281}$ & 0,054 \\
Textura del suelo & 0,084 & 0,016 & $\mathbf{0 , 9 1 7}$ & 0,012 \\
pH & 0,016 & $\underline{-0,343}$ & $\underline{0,306}$ & $\mathbf{0 , 5 4 4}$ \\
Actividad ureasa & $-0,023$ & $\mathbf{- 0 , 7 0 6}$ & $\underline{0,211}$ & $-0,008$ \\
Actividad catalasa & 0,080 & 0,103 & $-0,113$ & $\mathbf{0 , 8 8 7}$ \\
Valor propio & 1.93 & 1.33 & 1.03 & 1.00 \\
Varianza total (\%) & 27.5 & 19.1 & 14.7 & 13.9 \\
Varianza acumulada (\%) & 27.5 & 46.6 & 61.4 & 75.2 \\
\hline
\end{tabular}

Números en negrita corresponden a las principales correlaciones entre la variable y un factor latente específico. Números subrayados, indican una correlación secundaria, sin embargo, significativa de una variable con un factor latente específico. Fuente: autores

\subsection{DISCUSIÓN}

El objetivo de este estudio fue determinar la estructura multivariada de variables climáticas y edáficas en sistemas agropecuarios colombianos, mediante la técnica de AMFM. Los resultados muestran que variables como temperatura, altitud, humedad relativa, textura del suelo, $\mathrm{pH}$, actividad ureasa y actividad catalasa, se pueden categorizar en cuatro factores con significado edafoclimático.

Los presentes resultados indican, que, en la región geográfica evaluada (Figura 1), predominan suelos franco arcillosos y francos arenosos, con un pH entre 5,9 y 10,9, y que además presentan principalmente, actividades catalasa y ureasa bajas y medias. Esta caracterización inicial se constituye como una fuente de información importante para el productor de dicha región, debido a que existe una estrecha relación entre las características del suelo y las actividades agrícolas (Estupiñán-Gómez et al., 2009; Villaseñor et al., 2015), como también, por su relación con las prácticas ganaderas (Vallejo-Quintero, 2013); esto último, porque las características del suelo, en asocio con las condiciones climáticas, definen el tipo de praderas a ser cultivadas $y$, por ende, definen en parte, la producción animal.

El AMFM fue capaz de extraer cuatro factores con significado edafo-climático. El patrón de los factores (correlaciones entre cada factor y las variables originales) fue fácil de leer e interpretar. En particular, se observó después de examinar los patrones, que cada variable tenía una única y fuerte correlación superior a 0.5, con un único factor. Este tipo de estructura es un indicador de la viabilidad del AMFM para analizar la base de datos usada en este estudio (Johnson y Wichern, 2014).

Debido a que el análisis multivariado factorial es de carácter mixto, esto es, involucra variables cualitativas y cuantitativas, no fue posible calcular el índice Kaiser-Meyer-Olkin. Sin embargo, el valor bajo del logaritmo en 
base 10 del determinante de la matriz de correlaciones $(-0.60)$ para el presente análisis, indica que existen variables con inter-correlación altas y, en consecuencia, es factible la realización del análisis factorial.

Se observó que el primer factor latente, denominado como "Climático", está fuertemente asociado con las variables temperatura y altitud. Esto se puede deber, al alto y significativo coeficiente de correlación (R) que existe entre estas dos variables (Figura 2). El entendimiento desde un punto de vista cuantitativo de la asociación entre temperatura del ambiente y altitud, puede ser útil para el productor, debido a que estos son dos parámetros que influencian significativamente la fisiología de plantas y animales $y$, por ende, de los sistemas agropecuarios, como lo muestra el trabajo de Pineda-Santos y Suarez-Hernández, 2014), los cuales sugieren que tanto la temperatura del ambiente como la altitud, son dos variables fundamentales para la zonificación agroecológica de cultivos y el de Lozano (2017), quien demostró que las dos variables en mención tienen un impacto significativo en el desempeño biológico de plantas, en la zona alto-andina de Perú.

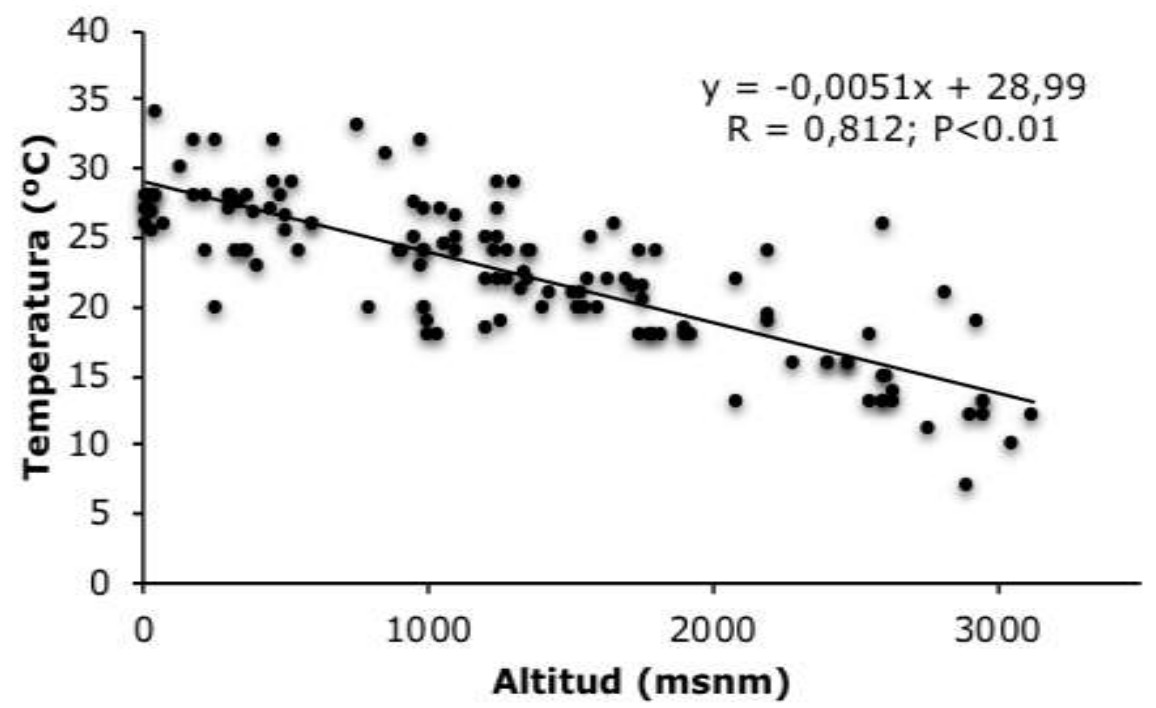

Figura 2. Regresión linear entre la altitud y temperatura de las propiedades rurales analizadas. Fuente: autores

De manera semejante, temperatura del ambiente y altitud, pueden afectar la producción de forraje, influyendo indirectamente la producción animal. Escobar (2018) demostró que la altitud influencia notoriamente la producción de pasto kikuyo (Cenchrus clandestinus Hochst ex Chiov) en la Sabana de Bogotá. Complementariamente, Wilson-García et al. (2017), revelaron que la temperatura del ambiente afectó significativamente el crecimiento de tres líneas de cebada, para la producción de forraje, en el valle de México - Texcoco. Por tanto, el AMFM determinó adecuadamente y con significado biológicamente coherente, la relación multivariada y cuantitativa existente entre temperatura del ambiente y altitud en los sistemas de producción agropecuarios colombianos. 
El segundo factor latente, denominado como Ureasa-agua-pH se asoció fuertemente con la humedad relativa y la actividad ureasa, y tuvo también una asociación intermedia con el $\mathrm{pH}$ del suelo. El entendimiento de la dinámica de los sistemas enzimáticos que componen el suelo es fundamental para establecer estrategias viables para su manejo. Algunos de estos sistemas, cumplen papeles importantes en procesos tales como la mineralización, movilización de nutrientes y fijación biológica de nitrógeno entre otros (Henríquez et al., 2014). Este es el caso de la fijación de nitrógeno, donde la enzima ureasa cataliza la conversión de urea a amonio y dióxido de carbono o bicarbonato. Su proceso catabólico se describe en la Figura 3 (Rodríguez-Jiménez et al., 2016).

(a)
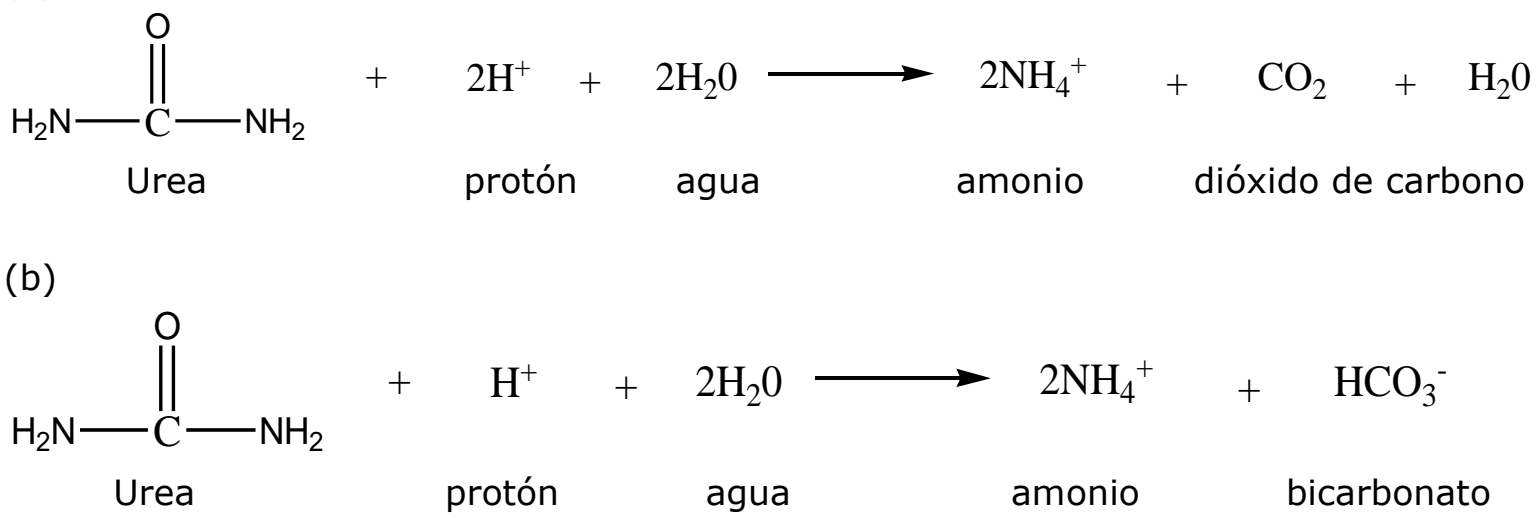

Figura 3. Proceso catalítico de la ureasa en suelo, cuando el pH es: (a) menor a 6,3; (b) mayor o igual a 6,3. Fuente: autores

De la figura 3, se puede observar que la cinética de la ureasa es afectada significativamente por el $\mathrm{pH}$ y el contenido de humedad del suelo, donde este último parámetro, es influenciado por la humedad relativa de ambiente (Ontiveros et al., 2016). La asociación entre estas variables es coherente con la estructura encontrada para el segundo factor, el cual está formado por las variables de humedad relativa y actividad ureasa, con una influencia del pH. Así las cosas, con este estudio, se demuestra que existe una fuerte asociación cuantitativa entre estas variables, en un sistema agropecuario. Esta información puede ser importante para el entendimiento del efecto de las condiciones climáticas en la fertilidad de suelos, considerando el papel fundamental de la ureasa en esta característica (Cerón y Melgarejo, 2005; Mogollón et al., 2016).

El tercer factor latente, denominado como "Fisicoquímica-suelo" está principalmente asociado a la variable textura del suelo. Sin embargo, también presentó una asociación parcial y significativa con las variables de humedad relativa, actividad ureasa y $\mathrm{pH}$. Esta estructura de variables para este factor se encuentra claramente de acuerdo con la literatura, ya que según Cerón y Melgarejo, 
(2005), tanto la textura del suelo como su actividad ureasa y $\mathrm{pH}$, son parámetros de calidad e indican el "status" biológico del suelo, y según, García et al. (2016), el contenido de humedad del suelo (correlacionado fuertemente a la humedad relativa), tiene un impacto en su textura. Así las cosas, evidentemente, las variables mencionadas, al ser parámetros de calidad en el suelo, no son independientes, lo cual se evidenció en el presente AMFM.

La catalasa es una enzima oxido-reductasa, que cataliza la descomposición de peróxido de hidrógeno $\left(\mathrm{H}_{2} \mathrm{O}_{2}\right)$ en oxígeno y agua. El $\mathrm{H}_{2} \mathrm{O}_{2}$ se genera durante los procesos respiratorios de los microorganismos aeróbicos en el suelo y este tiende a interactuar con la materia orgánica. Por tanto, la actividad catalasa, es un indicativo del nivel de materia orgánica y carga de microorganismos del suelo (Gili et al., 2014). Complementariamente, el pH tiene una influencia directa en la biodisponibilidad de diversos elementos y compuestos inorgánicos en el suelo, los cuales son vitales en procesos metabólicos asociados a la degradación y biosíntesis de materia orgánica en el suelo por parte de los microorganismos (Cerón y Melgarejo, 2005). De esta forma, podría esperarse una asociación fuerte entre la actividad catalasa y el $\mathrm{pH}$ en suelo, lo cual se evidenció en este estudio, al AMFM revelar un cuarto factor latente, compuesto por la actividad catalasa y el $\mathrm{pH}$ en suelo. De esta forma, el presente estudio brinda bases no solo cualitativas sino también cuantitativas viables, que podrían ser utilizadas para entender los mecanismos bioquímicos de interrelación, entre la actividad catalasa y ureasa, como también el impacto de estas, en la dinámica de crecimiento de microorganismos y producción de materia orgánica en el suelo del territorio colombiano.

\section{CONCLUSIONES}

De esta forma, del presente estudio se puede concluir que el análisis multivariado factorial mixto, reveló la estructura multivariada de variables climáticas y edáficas de interés para los sistemas de producción agropecuarios colombianos. El análisis reveló que las variables temperatura y altitud, pertenecen a un primer factor denominado como "Climático". Adicionalmente, que las variables humedad relativa y actividad ureasa, pertenecen a un segundo factor, denominado como "Ureasa-agua-pH". Sin embargo, estas últimas, también presentaron una asociación significativa con el tercer factor, denominado "Fisicoquímica-suelo", el cual está compuesto principalmente por la variable textura del suelo. Adicionalmente, el análisis reveló la existencia de un cuarto factor, denominado como "Catalasa-pH", el cual está compuesto de las variables actividad catalasa y $\mathrm{pH}$. Sin embargo, la variable $\mathrm{pH}$, presentó también una notable correlación con el factor Ureasaagua-pH.

De esta forma, las relaciones cuantitativas entre variables halladas por este estudio pueden ser de valor para aumentar el entendimiento de diversos factores físicos, químicos y microbiológicos que afectan la fertilidad y calidad de los suelos colombianos, información que puede ser útil para el diseño estrategias viables y eficientes para maximizar la producción animal y vegetal en los sistemas agropecuarios de la región en estudio. Adicionalmente, el presente trabajo deja en evidencia la viabilidad del análisis multivariado factorial mixto para entender diversas asociaciones complejas entre variables que hacen parte de sistemas agropecuarios, lo que viabiliza su aplicación futura en estudios adicionales que busquen entender tales relaciones en otros sistemas 
geográficos diferentes al estudiado y que a su vez, involucren un mayor número de variables cualitativas y cuantitativas.

\section{CONTRIBUCIÓN DE LA AUTORÍA}

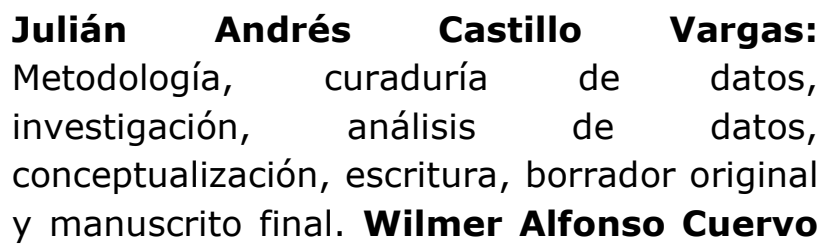

Vivas: Curaduría de datos, revisión de borrador original y manuscrito final.

\section{AGRADECIMIENTOS}

Los autores agradecen a la Universidad Nacional Abierta y a Distancia por el suministro de los datos para la realización de este estudio. Adicionalmente, a todos los estudiantes del curso de bioquímica metabólica, entre los años 2017 y 2018, de los cursos de Agronomía y Zootecnia, por contribuir con las informaciones usadas en este estudio.

\section{REFERENCIAS}

AccuWeather Inc. (2021). Recuperado de https://www.accuweather.com/

Agnusdei, M. G. (2013). Rol de la ecofisiología en el diseño de manejos especializados de pasturas. Archivos Latinoamericanos de Producción Animal, 21(1), 63-78.

Arreaza, L., García, S., Leal, J., Roncallo, B., y Mateus, H. (2012). Guía para la suplementación energético - proteico de bovinos en el trópico En: Alternativas Tecnológicas para la producción competitiva de leche y carne en el trópico bajo. Memorias Seminario CORPOICA, 2940.

Cerón, L. E., y Melgarejo, L. M. (2005). Enzimas del suelo: indicadores de salud y calidad. Acta Biológica Colombiana, 10(1), 5-18.

CSP. (2021). Climate Services Partnership. Recuperado de www.climate-services.org/

Escobar, M. A. (2018). Efecto de la madurez del pasto Kikuyo (Cenchrus clandestinus Hochst. ex Chiov.) sobre la producción de biomasa y la composición nutricional en diferentes altitudes de la provincia de Ubaté (tesis de maestría). Universidad Nacional de Colombia, Bogotá, Colombia.

Estupiñán-Gómez, L. H., Gomez, J. E., Barrantes, V. J., y Limas, L. F. (2009). Efecto de actividades agropecuarias en las características del suelo en el páramo El Granizo, (Cundinamarca - Colombia). Revista UDCA Actualidad \& Divulgación Científica, 12(2), 79-89.

Gaete, H., Hidalgo, M. E., Neaman, A., y Ávila, G. (2010). Evaluación de la toxicidad de cobre en suelos a través de biomarcadores de estrés oxidativo en Eisenia foetida. Química Nova, 33(3), 566 $-570$.

García, G., Brogioni, M., Venturini, V., Rodriguez, L., Fontanelli, G., Walker, E., Macelloni, G., y Graciani, S. (2016). Determinacion de la humedad de suelo mediante regresion lineal multiple con datos TerraSAR-X. Revista de Teledetección, 46, 73-81.

Gili, P., Marando, G., Irisarri, J., y Sagardoy, M. (2014). Actividad biolgica y enzimática en suelos afectados por sales del Alto Valle de Ro Negro y Neuquén. Revista Argentina de Microbiologia, 36, 187-192.

Hair, J. F., Black, W. C., y Babin, B. J. (2014). Anderson, R. E. Multivariate data analysis, Edinburg, UK: Pearson Education Limited.

Henríquez, C., Uribe, L., Valenciano, A., y Nogales, R. (2014). Actividad enzimática del suelo -Deshidrogenasa, $\beta$-Glucosidasa, Fosfatasa y Ureasa- bajo diferentes cultivos. Agronomía Costarricense, 38(1), 43-54. 
IDEAM. (2021). Instituto de Hidrología, Meteorología y Estudios Ambientales. Recuperado de www.ideam.gov.co/

Johnson, R. A., y Wichern, D.W. (2014). Applied multivariate statistical analysis, Edinburg, UK: Pearson Education Limited.

Kaiser, H. F. (1958). The varimax criterion for analytic rotation in factor analysis. Psychometrika, 23, 187-200.

Lozano, A. L. C. (2017). Desempeño de la planta Lepidium meyenii Walp. "maca" en parcelas experimentales localizadas en diferentes altitudes: la influencia del suelo y la procedencia de las semillas (tesis de pregrado). Universidad Peruana Cayetano Heredia, San Martín de Porres, Perú.

Mogollón, J. P., Martínez, A., y Torres, D. (2016). Efecto de la aplicación de vermicompost en las propiedades biológicas en un suelo salino-sódico del semiárido venezolano. Bioagro, 28(1), 2938.

Ontiveros, S., Manrique, S., y Franco, J. (2016). Flujos de metano en suelos forestales, dinámica y relación con factores edáficos y ambientales. XXXIX Reunión de Trabajo de la Asociación Argentina de Energías Renovables y Medio Ambiente (ASADES), La Plata, Argentina.

Pineda-Santos, L. D., y Suárez-Hernández, J. E. (2014). Elaboración de un SIG orientado a la zonificación agroecológica de los cultivos. Revista Ingeniería Agrícola, 4(3), 28-32.
Rodríguez-Jiménez, T. J., Ojeda-Barrios, D. L., Blanco-Macías, F., Valdez-Cepeda, R. D., y Parra-Quezada, R. (2016). Urease and nickel in plant physiology. Revista Chapingo Serie Horticultura, 22(2), 69-82.

Snedecor, G. W., y Cochran, W. G. (1989). Statistical methods, Iowa, US: Iowa State University Press.

USDA. (1999). Guía para la Evaluación de la Calidad y Salud del Suelo. Recuperado de https://www.nrcs.usda.gov/Internet/FSE DOCUMENTS/stelprdb1044786.pdf

Vallejo-Quintero, V. E. (2013). Importancia y utilidad de la evaluación de la calidad de suelos mediante el componente microbiano: experiencias en sistemas silvopastoriles. Colombia Forestal, 16(1), 83-99.

Villaseñor, D., Chabla, J., y Luna, E. (2015). Caracterización física y clasificación taxonómica de algunos suelos dedicados a la actividad agricola de la provinica del El Oro. Cumbres, 1(2), 28-34.

DOI: $10.14483 /$ udistrital.jour.colomb.for.2 013.1.a06

Wilson-García, C. J., Hernández-Garay, A., Ortega-Cerrilla, M. E., López-Castañeda, C., Bárcena-Gama, R., Zaragoza-Ramírez, J. L., y Aranda-Osorio, G. (2017). Análisis del crecimiento de tres líneas de cebada para producción de forraje, en el valle de México. Revista de la Facultad de Ciencias Agrarias de la Universidad Nacional de Cuyo, 49(2), 79-92.

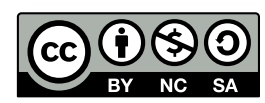

Licencia de Creative Commons

Revista Agricolae \& Habitat is licensed under a Creative Commons Reconocimiento-NoComercial-CompartirIgual 4.0 Internacional License. 
